\title{
3D culture of murine adipose-derived multipotent mesenchymal stromal cells in hydrogel based on carbomer 974P
}

\author{
Kyryk V. M. ${ }^{1}$, Kuchuk O. V. ${ }^{1}$, Mamchur A. A. ${ }^{1}$, Ustymenko A. M. ${ }^{1}$, Lutsenko T. M. ${ }^{1}$, Tsupykov O. M. ${ }^{1,2}$, \\ Yatsenko K. V. ${ }^{2}$, Skibo G. G. ${ }^{1,2}$, Bilko D. I. ${ }^{3}$, Bilko N. M. ${ }^{3}$ \\ ${ }^{1}$ State Institute of Genetic and Regenerative Medicine National Academy of Medical Sciences of Ukraine, Kyiv, Ukraine \\ ${ }^{2}$ Bogomoletz Institute of Physiology National Academy of Sciences of Ukraine, Kyiv, Ukraine \\ ${ }^{3}$ Center of molecular and cell research of National University of Kyiv-Mohyla Academy of Ministry of Education and Science of Ukraine, \\ Kyiv, Ukraine \\ e-mail: labcellculture@gmail.com
}

\section{ABSTRACT}

Actual issues during tissue regeneration are to ensure the survival of transplanted cells at the site of their application and further activity, especially in case of local pathological alterations such as inflammation and ischemia. For this purpose, the matrices that can not only fill the defects of tissues, but also be scaffolds for cells are developed.

THE AIM of this study was to evaluate the effectiveness of 3D cultivation of murine adipose-derived multipotent mesenchymal stromal cells (MSMCs) in hydrogel based on carbomer 974P.

MATERIALS AND METHODS. MSMCs were obtained from the adipose tissue of FVB-Cg-Tg(GFPU)5Nagy/J mice transgenic for GFP gene. The cells were phenotyped by flow cytometry and directly differentiated into osteogenic and adipogenic direction to confirm multipotent phenotype. MMSCs were cultured and directly differentiated into osteogenic direction in three-dimensional hydrogel scaffolds. For hydrogel preparation we used carbomer 974P with composition of glycerol, propylene glycol, triethylamine and agarose in original proportion.

RESULTS. The three-dimensional hydrogel based on carbomer 974P for the further engraftment with MMSCs was obtained. Modified protocols for the preparation of hydrogels based on carbomer and agarose and their rehydration by culture media for the 3D cultivation of adiposederived MMSCs have been developed. The optimal concentration of MSMCs and the injection method for engraftment of hydrogels of the required form and size are selected. It was shown that adipose-derived MMSCs in 3D carbomer hydrogel preserve the potential of directed osteogenic differentiation.

CONCLUSION. Three-dimensional hydrogel based on carbomer 974P is capable to support cells, provide the necessary cytoarchitectonics, maintain intercellular interactions, which can promote further long-term survival and specialization of the graft.

KEY WORDS: adipose-derived multipotent mesenchymal stromal cells; 3-dimentional cell culture; hydrogel; carbomer 974P

Nowadays cell and tissue technologies are the most promising method for treatment of various diseases instead of existing surgery approaches and drug therapy if well-known techniques are being demonstrated as less effective. Stem cell transplantation cannot only confer tissue regeneration; it can also facilitate endogenous repair process due to the host progenitor cells activation. However, the most important problem in tissue and organ reparation is a long-term engraftment of transplanted cells at target sites for further effective activity especially in case of pathological alterations of the injury site as an ischemia and inflammation [1].
The method of cell culturing is widely used in the basic research, biotechnology industry and in the clinics towards regenerative medicine. The majority of cell culture studies have been performed on 2D culture systems due to the simplicity of method and high cell viability [2]. The growth of cells as 2D monolayers has notably improved the understanding of cellular biology. However, almost of all cells in organism are surrounded by extracellular $3 \mathrm{D}$ matrix that provides complex biochemical and physical signals between cells of different types [3]. Thus, recently it has become more and more relevant to develop 3D cell culture systems 
over the variety of matrixes. At present, more than 380 cell lines have been tested for their potential to form and maintain 3D spheroids [4].

It is shown in vitro that $3 \mathrm{D}$ spheroids of MMSCs produced higher levels of extracellular matrix proteins (including collagen I, fibronectin and laminin), compared with $2 \mathrm{D}$ cultured cells. Additionally, 3D culture increased the paracrine secretion of cytokines, including angiogenic factors (VEGF and bFGF), anti-apoptotic factors (epidermal growth factor and hepatocyte growth factor), the anti-oxidative factors (IGF, TNF-a) [5]. It is also known that $3 \mathrm{D}$ spheroids allow MMSCs to keep the multipotency, which does not occur in $2 \mathrm{D}$ cell culture [6]. The adoption of spherical shape could induce a rearrangement of cytoskeleton, potentially involved in regaining in earlier stages of potency via E-cadherin-mediated mechanism as well as influence on physical properties of cell stemness [7].

To modify a proper cytoarchitectonics in 3D systems, the next common characteristics should be considered: mechanical support, chemical compounds, cell grow stimulation and signaling, transportation of nutrients, gases and cell metabolic products [8]. There were a dozen types of scaffolds suggested by different biotechnological industries for the purpose of regenerative medicine over last years. The origin of platform, method of obtaining, physical and chemical properties, biological inertness, and ability to resorption are between advantages and disadvantages of matrix application. There are known more than 100 different matrixes both organic (collagen, gelatin, elastin, chitosan, chitin, fibrin, fibrinogen etc.) and non-organic origin $[9,10,11]$. Natural and plastic based materials are used as a background for 3D scaffolds [12]. Thus, such materials provide the proper extracellular matrix due to their structure (porosity, stringiness, permeability) and mechanical support. These scaffolds are appropriate for the mechanical support or biodegradation and can be used as stable transplants. 3D platforms provide biologically active environment and subsequent better biophysical and biochemical cell-cell interaction, proliferation, differentiation and extracellular matrix secretion [13].

Different types of hydrogel are applied as a basement for the 3D scaffolds in biomedicine. The hydrogels made from naturally occurred biopolymers (such as alginate and gelatin) are reliable materials due to their biocompatibility and potency to the biodegradation [14, 15]. Challenges in applying hydrogels for 3D tissue constructs are the control of cell material interaction and realization of inner space to allow cell ingrowth and communication. Furthermore, a challenge in constructing 3D hydrogel tissue scaffolds is the high total porosity (more $60 \%$ ), which influences on the cell seeding effectiveness $[16,17]$.

One of the promising options for creating a 3-dimensional matrix is hydrogels based on agarose and carbomer. Carbomer or Carbopol is a polyacrylicacid polymer, which shows a sol to gel transition in aqueous solution as the $\mathrm{pH}$ is raised above its pKa of about 5.5 [18]. Due to their properties, carbomer hydrogels realize the potential of scaffolds of natural origin, providing a 3D structure similar to living tissue, while remaining non-toxic and non-immunogenic [19]. On the other hand, due to the ability to regulate the physical and chemical parameters of these hydrogels, changing their proportions and modifications of the components, the necessary kinetics of degradation of the scaffold is achieved, which correlates with the integration of the graft into the recipient's tissues [20].

The aim of this study was to evaluate the effectiveness of 3D cultivation of murine adipose-derived multipotent mesenchymal stromal cells in hydrogel based on carbomer 974P.

\section{MATERIALS AND METHODS}

In our study we used FVB-C-Tg (GFPU) 5Nagy/J mice transgenic for green fluorescent protein (GFP) kindly provided by European Molecular Biology Laboratory, Monterotondo, Italy. The animals were kept under standard conditions at the vivarium of State Institute of Genetic and Regenerative Medicine with free access to food and water ad libitum. All experiments were performed in compliance with international principles of the European Convention for the Protection of vertebrate animals used for experimental and other scientific purposes (European convention, Strasburg, 1986), Article 26 of the Law of Ukraine «On protection of animals from cruelty» (No. 3447-IV, 21.02.2006) and all norms of bioethics and biosafety.

\section{ISOLATION OF ADIPOSE-DERIVED MIMSCS.}

The 4-month-old female mice $(n=3)$ were euthanized by cervical dislocation under ether anesthesia. Under sterile conditions inguinal adipose tissue was incised, minced with scissors into fragments $1 \times 1 \mathrm{~mm}$ then treated with $0.1 \%$ collagenase type I in DMEM medium (Sigma, USA) for $90 \mathrm{~min}$ at $37^{\circ} \mathrm{C}$ on shaker. After washing and filtration trough 70 $\mu \mathrm{m}$ strainer, cell suspension was cultured in a $25 \mathrm{~cm}^{2}$ flask in complete DMEM culture medium supplemented with $15 \%$ fetal bovine serum FBS (HyClon, USA), penicillin $100 \mathrm{U} / \mathrm{ml}$, streptomycin $100 \mu \mathrm{g} / \mathrm{ml}, 1: 100$ nonessential amino acids (all - Sigma-Aldrich, USA) in a $\mathrm{CO}_{2}$-incubator $\left(37{ }^{\circ} \mathrm{C}, 5 \% \mathrm{CO}_{2}\right.$, humidified atmosphere). When culture reached $80 \%$ confluency, cells were subcultured using $0.25 \%$ trypsin. Cells of 2 passages were used for hydrogel engraftment.

The phenotyping of cell cultures for CD44, CD73, CD90, CD45, CD117, and CD34 markers was performed using FACSAria cell sorter (Becton Dickinson, USA). Samples of $2 \cdot 10^{5}$ cells in $50 \mu$ medium were incubated with fluorochrome-conjugated monoclonal antibodies anti-CD44-PE, anti-CD73-PE, anti-CD90-PE, anti-CD45-PE, anti-CD117-PE-Cy7, and anti-CD34-APC (BD Bioscience, USA) in a working concentration of 0.5 $\mu \mathrm{g} / \mathrm{ml}$ for 30 min then were washed in CellWash (BD Bioscience, USA) by centrifugation at $300 \times \mathrm{xg}$ for $5 \mathrm{~min}$. At least $2 \cdot 10^{4}$ cells per sample were recorded for analysis using BD FACSDiva 6.2.1 software. To compensate fluorochrome overlap for multiparameter analysis the control samples of cells without antibodies (unstained control), samples with each antibody alone (single stained control) and samples with a combination of several antibodies without one (fluorescence minus one - FMO control) were used [21]. Cell viability was determined using 7-AAD staining (BD Bioscience, USA).

\section{DIRECTED DIFFERENTIATION OF ADIPOSE-DERIVED MIMISCS}

Osteogenic cell differentiation was induced by modified methods $L i$ et al. [22]. At the second passage, culture medium was changed to medium for osteogenic differentiation which consisted of DMEM-F12 medium (Sigma-Aldrich, USA) supplemented with $10 \%$ FBS (HyClon, USA), and contained $0.05 \mathrm{mM}$ L-ascorbic acid 2-phosphate, $100 \mathrm{nM}$ dexamethasone and $10 \mathrm{mM} \beta$-glycerophosphate (all - Sigma-Aldrich, USA). Osteogenic medium was changed every 3 days. After 21 days under standard conditions in a $\mathrm{CO}_{2}$ incubator cell monolayer was washed with phosphate buffered saline (PBS), fixed with cold $4 \%$ paraformaldehyde for 25-30 min than stained with Alizarin Red S (Sigma-Aldrich, USA) to detect calcium phosphate salts deposit in extracellular matrix which is one of the signs of osteogenic differentiation. In addition, the production of alkaline phosphatase was detected by BCIP/NBT staining (Sigma-Aldrich, USA) for 20-30 min in the dark. After that cells were washed with distilled water and intensity of staining was analyzed [23].

The adipogenic differentiation was induced in DMEM-HG medium (Sigma-Aldrich, USA) supplemented with $10 \%$ FBS (HyClon, USA) containing $1 \mu \mathrm{M}$ dexamethasone, $200 \mu \mathrm{M}$ indomethacin, $500 \mu \mathrm{M}$ isobutylmethylxanthine and $5 \mu \mathrm{g} / \mathrm{mL}$ insulin (all - Sigma-Aldrich, USA) [24]. The adipogenic medium was changed every 3 days for 14 days in culture. Cells were fixed in cold $4 \%$ paraformaldehyde and stained with Oil Red 0 (Sigma-Aldrich, USA) to detect lipid droplets in the cytoplasm.

\section{HYDROGEL PREPARATION AND CELL CULTURE IN HYDROGELS}

For hydrogel preparation we used carbomer 974P combined with glycerol, propylene glycol, triethylamine and agarose in original proportion. Gel was heated to $83-85^{\circ} \mathrm{C}$ and placed in plastic forms of necessary sizes (10 mm diameter, $5 \mathrm{~mm}$ high) with subsequent cooling to room temperature. Prepared scaffolds were lyophilized and sterilized under UV in a biosafety cabinet. 
The hydrogels were rehydrated in complete culture medium, than MMSCs were seeded using pipette tip $(n=6)$ or injected by insulin syringe $(n=10)$ all over the surface of the scaffold at $4 \cdot 10^{4}, 2 \cdot 10^{5}$ and $1 \cdot 10^{6}$ cells per each hydrogel. Cultivations were performed under standard conditions in a DMEM medium supplemented with $10 \%$ FBS. The culture medium was replaced every 3-4 days.

\section{DIRECTED OSTEOGENIC DIFFERENTIATION OF ADIPOSE-DERIVED MIMSCS IN CARBOMER HYDROGEL}

On the $14^{\text {th }}$ day of cultivation of hydrogels engrafted with $1 \cdot 10^{6}$ cells $(n=4)$, the complete culture medium was changed with an appropriate medium for osteogenic induction. Cells were cultured under osteogenic conditions for 14 days, the medium was changed every 3-4 days.

To evaluate the effectiveness of osteogenic induction, control and experimental samples of hydrogels were fixed in a $4 \%$ solution of paraformaldehyde in $0.1 \mathrm{M}$ phosphate buffered saline for 48 hours at $+4{ }^{\circ} \mathrm{C}$ and stained with Alizarin Red $\mathrm{S}$ to detect calcium salts. The production of alkaline phosphatase was detected by BCIP/NBT staining.

Monolayer cultures of cells and engrafted hydrogels were examined using an inverted fluorescence microscope IX-71 (Olympus, Japan) with a camera DP-20 (Olympus, Japan) using a QuickPHOTO software (Promicra, s.r.o., Czech Republic).

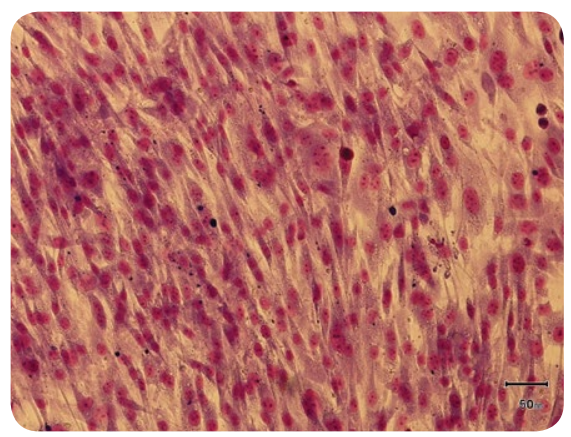

Fig. 1. Microphotograph of murine adiposederived stromal cells culture, $2^{\text {nd }}$ passage, light microscopy, azureeosin staining, scale bar $-50 \mu \mathrm{m}$.

\section{STATISTICAL ANALYSIS}

Statistical analysis was performed using Statistica v. 5 software (StatSoft Inc., USA). The results are presented as mean \pm standard error of mean (SEM).

\section{RESULTS AND DISCUSSION}

To confirm the proper characteristics of the adipose-derived MMSCs that were used for engraftment to the hydrogel, the obtained cultures were analyzed by morphology, immunophenotype and potential for directed differentiation into osteogenic and adipogenic direction [25].

In primary cultures, growth of spindle-shaped or polygonal cells with severe processes was observed. Further subcultures contributed to the formation of a more homogeneous population of fibroblast-like adhesive cells, which in a short time (2-3 days) doubled the population and formed a confluent monolayer. In some cases, growth was observed by increasing the cell colony size. Cell cytoplasm in this case was homogeneous, nuclei and nucleoli were clearly visualized (Fig. 1).

At the phenotyping of adipose-derived MMSCs from the initial to the second passages, there was an increase in the expression of the mesenchymal markers CD44 (up to $97.1 \pm 2.2 \%$ ), CD90 (up to $95.9 \pm 1.8 \%$ ) and CD73 (up to $64.3 \pm 14,6 \%$ ) had been noted, which corresponds to the typical MMSC phenotype (Fig. 2). At the same time, low expression $(<3 \%)$ of hematopoietic markers CD45 $(2.1 \pm 0.8 \%)$ and CD117 $(2.2$ $\pm 0.7 \%$ ) was observed. Expression of CD34 remained at $12 \pm 4 \%$ for the second passage. As it is generally known, the relatively high level of CD34 expression at the early passages is typical for the culture of adipose-derived MMSCs and may indicate a high potential of these cells for differentiation in the endothelial direction [26]. This feature of adiposederived multipotent cells can contribute to activation of neoangiogenesis and better vascularization of grafts to ensure their survival. Cell viability after subculture from monolayer into a suspension for further 3D culture determined by $7-A A D$ was $92.4 \%$.
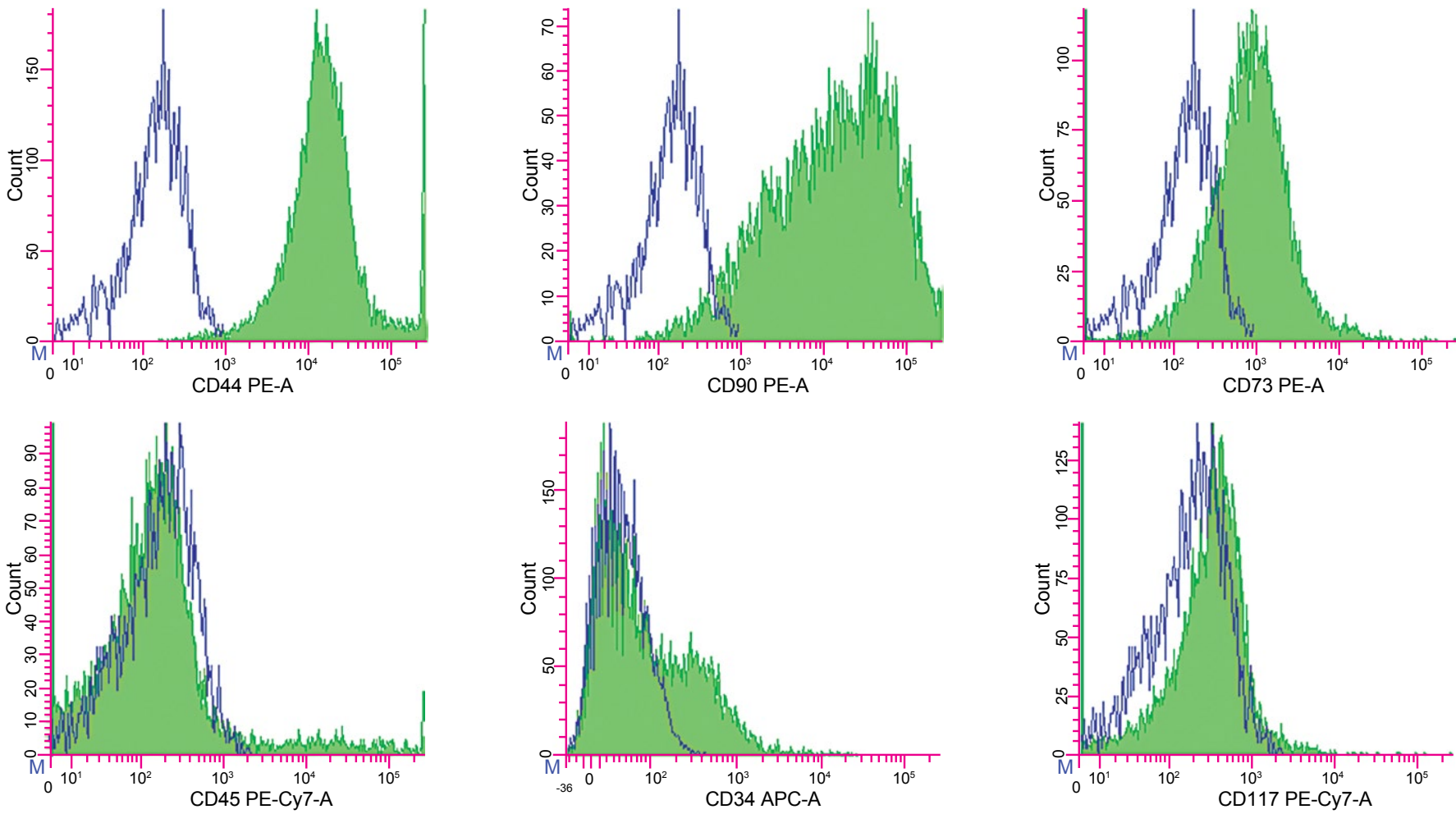

Fig. 2. Histograms of the expression of CD44, CD70, CD90, CD45, CD34 and CD117 markers in the culture of murine adipose-derived MSMC (green) compared to unstained controls (blue) according to flow cytometry, $2^{\text {nd }}$ passage, BD FACSDiva software. 

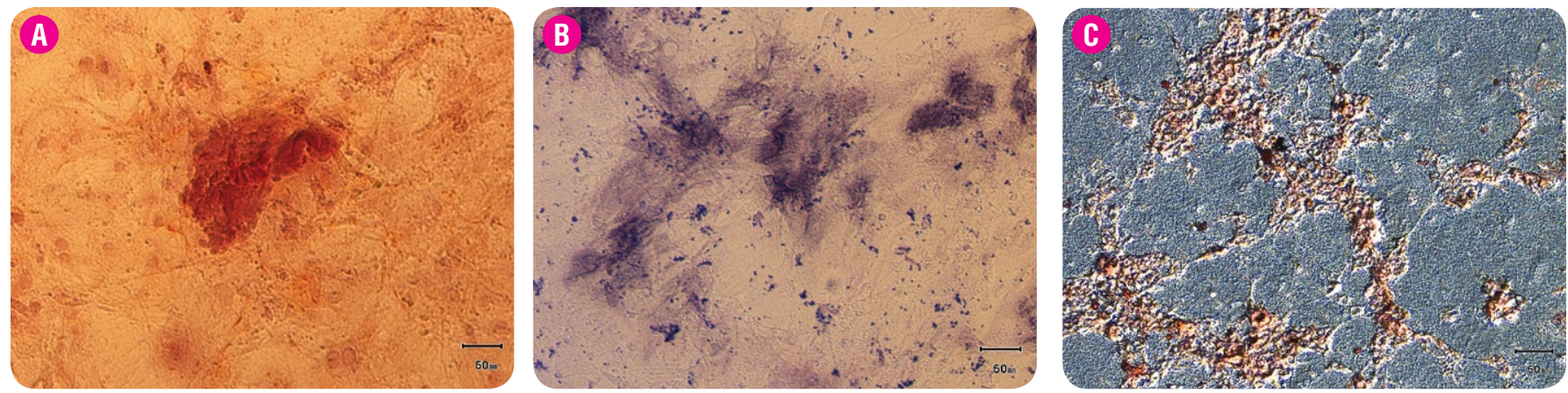

Fig. 3. Microphotographs of murine adipose-derived MMSCs, differentiated into the osteogenic direction (A-B), 21-day culture, staining with Alizarin Red S (A) and BCIP-NBT (B); and differentiated into the adipogenic direction (C), the $14^{\text {th }}$ day of cultivation, staining with 0 il Red 0 , scale bar - $50 \mu$ m.
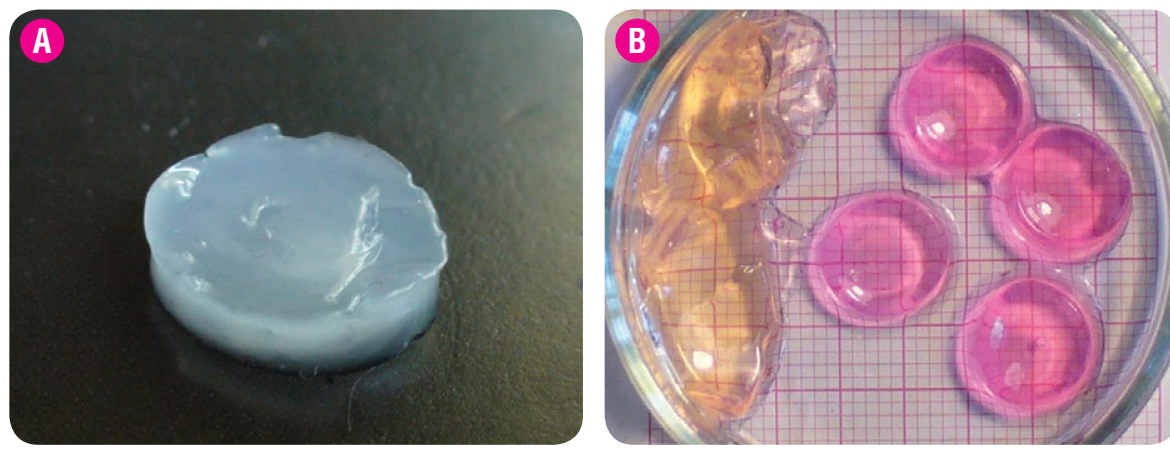

$<$

Fig. 4. Photo of hydrogels based on carbomer $974 \mathrm{P}$ and agarose: $\mathbf{A}-$ native hydrogel, B - hydrogels rehydrated with DMEM media.
The multipotent properties of the obtained murine adipose-derived MMSCs according to the ability to direct differentiation using histochemical staining for calcium salt deposits and alkaline phosphatase production at osteogenic induction or for lipid inclusions - at adipogenic differentiation were confirmed. On the $21^{\text {st }}$ day of osteogenic induction, in the adipose-derived MMSCs culture an intensive mineralization of the extracellular matrix with calcium salts was observed which was detected by staining with Alizarin Red S (Fig. $\mathbf{3 A}$ ), as well as high activity of alkaline phosphatase production, which was detected by BCIP-NBT staining (Fig. 3B). At the directed adipogenic differentiation after 14 days of cultivation in the appropriate medium, the presence of lipid droplets in the cytoplasm of the cells was detected (Fig. 3C).

Thus, according to morphological characteristics, immunophenotype and potential for directed differentiation, the obtained cultures of adiposederived cells that were used in subsequent experiments, met the minimum criteria for MMSCs provided by International Society for Cellular Therapy [25].

For three-dimensional cell culture, hydrogels based on agarose and carbomer 974P were made. Using the original proportions of the initial components and modifying the time and temperature of their processing, it is possible to achieve a certain plasticity of the material to provide it with the necessary form (Fig. 4A) and elastic properties with further rehydration of the nutrient medium (Fig. 4B).

We obtained 3D cultures of murine adipose-derived MMSCs engrafted in hydrogel rehydrated with nutrient medium. It was shown that in the second week when $4 \cdot 10^{4}$ cells were seeded to a cylindrical hydrogel with a diameter of $10 \mathrm{~mm}$ (volume $\sim 400 \mathrm{~mm}^{3}$ ), only single cells penetrated to a depth of $1.5 \mathrm{~mm}$ from the surface of the hydrogel and acquired an elongated shape. Most cells retained the rounded form and were on the surface of the hydrogel. When seeding by pipette tip of $10^{6}$ cells to a hydrogel in the early stages of cultivation (up to 10 days), the cells were located on its surface and kept round. During the next 5-7 days, the cells penetrated the gel and acquired an elongated shape, contacting with each other. After 7-14 days after penetration, the number of intercellular contacts increased and they formed clusters and a network of adjacent cells, indicating their proliferation (Fig. 5).
An injection method for engraftment of MMSCs into the carbomer hydrogel using a syringe was proposed and the dependence of its efficiency on the initial number of cells was analyzed. When we injected $4 \cdot 10^{4}$ or $2 \cdot 10^{5}$ cells into a hydrogel at a depth of $1-2 \mathrm{~mm}$, after 2 weeks in culture, the cells were located diffusively in the hydrogel, they acquired an elongated shape but did not contact with each other. When we injected $10^{6}$ cells into a hydrogel at a depth of 2-3 $\mathrm{mm}$ after 2 weeks of cultivation, the cells acquired polygonal form due to the numerous processes they contacted each other, forming a network of adjacent cells (Fig. 6).

Thus, a required concentration of cells and an optimal cell engraftment method for hydrogels of certain form and size for further in vitro studies were selected.

The method for directed osteogenic differentiation of adipose-derived MMSCs in carbomer hydrogel to assess their ability to multipotency under conditions of three-dimensional scaffold cultures have been developed. When hydrogels with cells in an osteogenic medium on the 14th day were stained with Alizarin Red S and BCIP-NBT dyes, a positive reaction on the calcium salt and alkaline phosphatase, respectively, on the periphery of the hydrogel and inside where cells localized was found (Fig. 7).

The obtained results indicate that adipose-derived multipotent mesenchymal stromal cells preserve the potential for directed differentiation under 3D culture in carbomer hydrogel, typical for two-dimensional monolayer cultures.

It is considered that regenerative potential of stem cells is realized with local cells environment (their niche), which provides direct cell-cell interaction and non-contact communications with the action of secreted signaling molecules of different type of cells [27]. The engineering of the stem cell microenvironment plays a crucial role in regenerative medicine and is considered as an approach to improve the regenerative potential of transplantation. [28]. For this purpose, culture matrixes are being developed. It is providing cells with the necessary tissue structure and reconstruction of the niche in vitro. [29]. The urgent task is to establish the influence of conditions of three-dimensional cultivation on the efficiency of survival, proliferation and potential for directed differentiation of multipotent stem cells of different origin. 

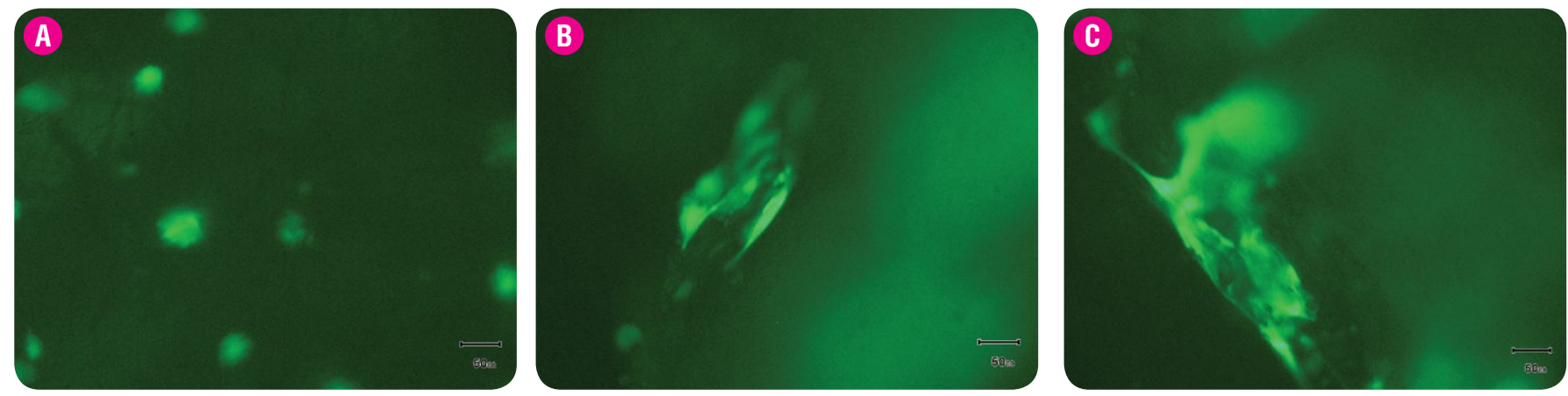

Fig. 5. Microphotographs of adipose-derived MMSCs in a carbomer hydrogel after $10^{6}$ cells seeding on hydrogel surface: $\mathbf{A}-8^{\text {th }}$ day of cultivation; B $-18^{\text {th }}$ day of cultivation; $\mathbf{C}-29^{\text {th }}$ day of cultivation, scale bar $-50 \mu \mathrm{m}$.
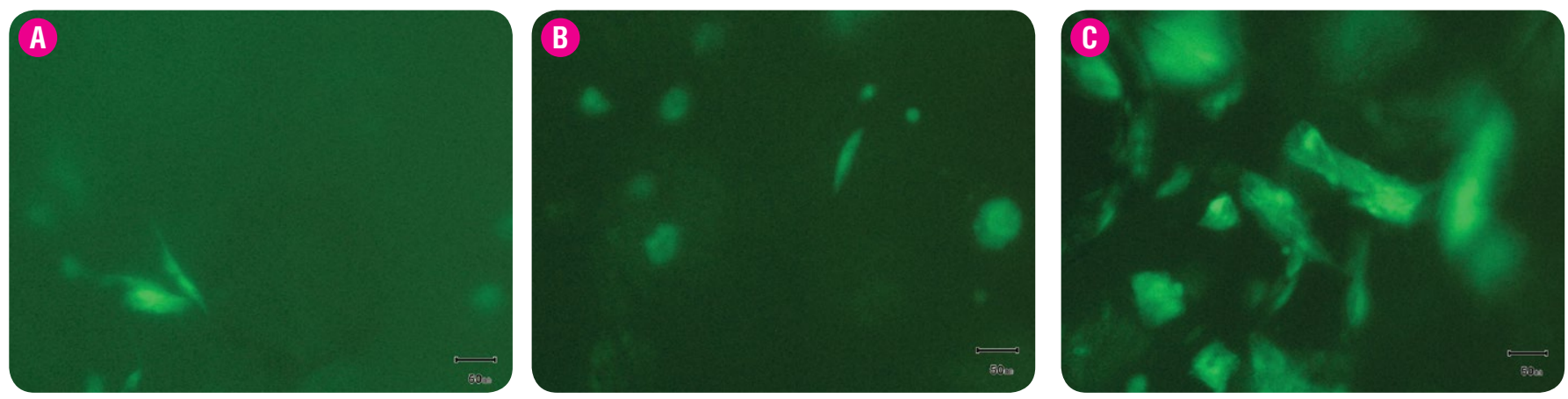

A Fig. 6. Microphotographs of adipose-derived MMSCs in a carbomer hydrogel after cell engraftment into hydrogel by microinjection, $14^{\text {th }}$ day of cultivation, fluorescence microscopy: A - initial dose of $4 \cdot 10^{4}$ cells; $\mathbf{B}-2 \cdot 10^{5}$ cells; $\mathbf{C}-10^{6}$ cells, scale bar $-50 \mu \mathrm{m}$.
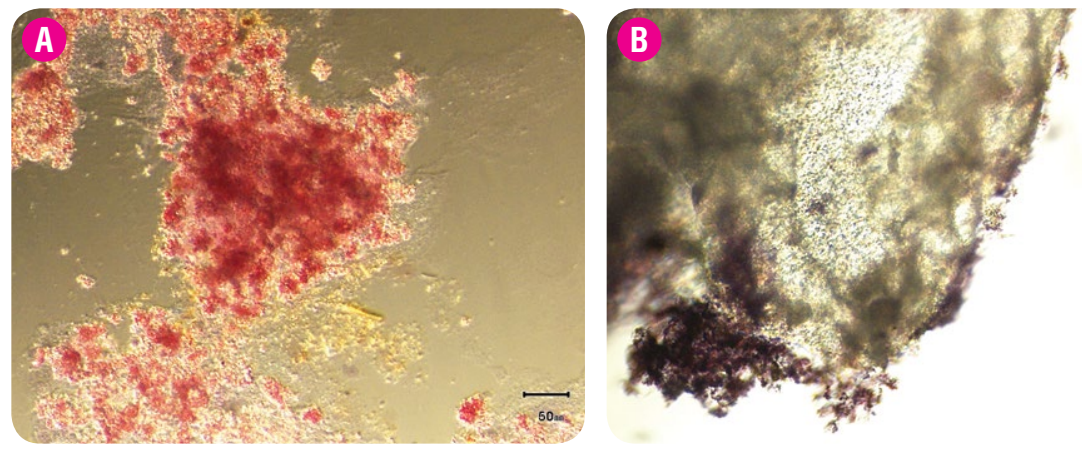

1

Fig. 7. Microphotographs of carbomer hydrogels fragments engrafted with murine adipose-derived MMSCs differentiated into osteogenic direction. Alizarin Red S staining for calcium phosphate salts

(A, red) and staining with BCIP-NBT for alkaline phosphatase (B, brown), scale bar $-50 \mu \mathrm{m}$.
In addition to form-forming and supporting functions, matrixes can also regulate growth factors releasing during different time points providing not only cell proliferation but their direct differentiation in vivo as well $[30,31]$. It is shown that cell-cell interaction with extracellular basement of biomaterial and growth factors provide stem cell differentiation and tissue growth [32, 33]. For example, bone marrow microenvironment factors (such as VEGF, TGF- $\beta 3$, BMP-2) increase the survival of adipose-derived stem cells in the recipient organism [34, 35]. In our previous studies for multipotent stromal cells from subcutaneous adipose tissue, the possibility of a stage-by-stage osteogenic differentiation with prior chondrogenic induction was demonstrated, which partially reproduces in vitro conditions of niche for the laying and formation of bone in the body [36]. The combination of technologies for the creation of 3D hydrogel scaffolds and the directed differentiation of the engrafted cells opens wide prospects for regenerative medicine. Step-by-step, directed differentiation of multipotent cells in vitro in three-dimensional constructions will allow partially reconstruct the physiological mechanisms of tissue formation in vivo and obtain a spatially organized cell culture capable of surviving, proliferating and differentiating in a required direction.

Fast and reliable cells seeded over 3D scaffolds require development of new biotechnological approaches using three-dimensional cul- ture model with subsequent evaluation of its safety and effectiveness, which is demonstrated in our study. The dynamic perfusion of transplants over bioreactor promotes 3D-matrix cell seeding and their directed induction. It is allowed to standardize the protocols of cell culture as well [37]. That is why further studies are needed to include in the composition of carbomer hydrogels different growth factors or cytokines responsible for chemotaxis, proliferation and tissue-specific differentiation of transplanted or endogenous stem cells.

Formed 3D scaffolds based on carbomer hydrogels can be used as transplants to study recovery processes at tissue injury in an in vivo experiments [19]. In general, the practical implications of this study will be the development of new biotechnological approaches for increasing effectiveness of adipose-derived MMSCs as an alternative source of adult stem cells in the injured ischemic tissue regeneration.

A dozen of the clinical application of matrixes within variety types of cells has been described in cases of bone, cartilage, intervertebral disk damages and skin injures. Furthermore, clinical trials were suggested to assess matrix efficacy and safety. These approaches are of the particular interest especially in reconstructive surgery in case of regeneration of soft tissues damages and wound healing [38, 39, 40]. 


\title{
CONCLUSION
}

Thus, three-dimensional hydrogels based on carbomer 974P are capable to mechanical support of cells growth providing the necessary cytoarchitectonics through the formation of intercellular contacts, and preserve the potential of multipotent cells for directed differentiation.

Improving the technology of cells engraftment in three-dimensional scaffolds based on the carbomer 974P will allow modeling their spatial organization and maintaining cell-cell interactions that can ultimately promote to the long-term survival and specialization of grafts for more effective repair of damaged tissues and organs.

\begin{abstract}
Acknowledgments
The research is carried out with support from the research project of Science and Technology Center in Ukraine № 6262 "Development of technology for three-dimensional culture of multipotent cells to regenerate tissue after ischemic injury». The authors wish to express their special appreciation to Dr. Giuseppe Perale, Politecnico di Milano (Italy) for his support and kind advice provided during this study.
\end{abstract}

\section{REFERENCES}

1. Mashkouri S, Crowley MG, Liska MG, Corey $S$, Borlongan CV. Utilizing pharmacotherapy and mesenchymal stem cell therapy to reduce inflammation following traumatic brain injury. Neural Regen Res. 2016; 11:1379-84.

2. Duval K, Grover H, Han LH, et al. Modeling physiological events in 2D vs. 3D cell culture. Physiology (Bethesda). 2017. 32(4):266-277.

3. Kim SH, Turnbull J, Guimond S. Extracellular matrix and cell signalling: the dynamic cooperation of integrin, proteoglycan and growth factor receptor. J Endocrinol. 2011; 209(2):139-51. DOI: 10.1530/JOE-10-0377.

4. Knight E, Przyborski S. Advances in 3D cell culture technologies enabling tissue-like structures to be created in vitro. J Anat. 2015; 227(6):746-756.

5. Feng G, Zhang J, Li Y, et al. IGF-1 C domain-modified hydrogel enhances cell therapy for AKI. J Am Soc Nephrol. 2016. 27(8):2357-2369.

6. Baraniak PR, Cooke MT, Saeed R, et al. Stiffening of human mesenchymal stem cell spheroid microenvironments induced by incorporation of gelatin microparticles. J Mech Behav Biomed Mater. 2012. 11:63-71.

7. Labusca LS. Scaffold free 3D culture of mesenchymal stem cells; implications for regenerative medicine. J Transplant Stem Cel Biol. 2015 ; 2(1):8-15.

8. Grigore A, Sarker B, Fabry B, et al. Behavior of encapsulated MG-63 cells in RGD and gelatine-modified alginate hydrogels. Tissue Eng. Part A. 2014. 20(1516):2140-2150.

9. Xing Q, Qian Z, Tahtinen M, et al. Aligned Nanofibrous Cell-Derived Extracellular Matrix for Anisotropic Vascular Graft Construction. Adv Healthc Mater. 2017; 6(10). DOI: 10.1002/adhm.201601333.

10. Capulli AK, MacQueen LA, Sheehy SP, Parker KK. Fibrous scaffolds for building hearts and heart parts. Adv Drug Deliv Rev. 2016; 15(96):83-102. D0I: 10.1016/j. addr.2015.11.020

11. Wu S, Duan B, Qin X, Butcher JT. Living nano-micro fibrous woven fabric/hydrogel composite scaffolds for heart valve engineering. Acta Biomater. 2017; 51 :89100. DOI: 10.1016/j.actbio.2017.01.051.

12. Ye H, Zhang K, Kai D, Li Z, Loh XJ. Polyester elastomers for soft tissue engineering. Chem Soc Rev. 2018; 47(12):4545-4580. D0I: 10.1039/c8cs00161h.

13. Alhaque $S$, Themis $M$, Rashidi $H$. Three-dimensional cell culture: from evolution to revolution. Philos Trans R Soc Lond B Biol Sci. 2018. 373(1750):20170216.

14. Tsou YH, Khoneisser J, Huang PC, Xu X. Hydrogel as a bioactive material to regulate stem cell fate. Bioact Mater. 2016; 1(1):39-55. D0I: 10.1016/j. bioactmat.2016.05.001.

15. Mei Liu, Xin Zeng, Chao Ma. Injectable hydrogels for cartilage and bone tissue engineering. Bone Res. 2017; 5:17014. D0I:10.1038/boneres.2017.14.

16. Sarker B, Rompf J, Silva R, et al. Alginate-based hydrogels with improved adhesive properties for cell encapsulation. Int J Biol Macromol. 2015. 78:72-78.

17. Ivanovska J, Zehnder T, Lennert P, et al. Biofabrication of 3D Alginate-Based Hydrogel for Cancer Research: Comparison of Cell Spreading, Viability, and Adhesion Characteristics of Colorectal HCT116 Tumor Cells. Tissue Eng Part C Methods. 2016; 22(7):708-15. D0l: 10.1089/ten.TEC.2015.0452.

18. Gupta S, Vyas SP. Carbopol/chitosan based pH triggered in situ gelling system for ocular delivery of timolol maleate. Sci Pharm. 2010; 78(4):959-976.

19. Hayati F, Ghamsari SM, Dehghan MM, Oryan A. Effects of carbomer 940 hydrogel on burn wounds: an in vitro and in vivo study. J Dermatolog Treat. 2018; 9(6):593-599. DOI: 10.1080/09546634.2018.1426823

20. Rossi F, Santoro M, Casalini T, et al. Characterization and Degradation Behavior of Agar-Carbomer Based Hydrogels for Drug Delivery Applications: Solute Effect. Int J Mol Sci. 2011; 12(6):3394-3408. D0I:10.3390/ijms12063394.

21. Maecker HT, Trotter J. Flow cytometry controls, instrument setup, and the determination of positivity. Cytometry A. 2006; 69(9):1037-42.

22. Li J, Mareddy S, Meifang DT, et. al. A minimal common osteochondrocytic differentiation medium for the osteogenic and chondrogenic differentiation of bone marrow stromal cells in the construction of osteochondral graft. Tissue engineering. 2009; Part A. 15(9):2481-90.

23. Mishra R, Kumar A. Osteocompatibility and osteoinductive potential of supermacroporous polyvinyl alcohol-TEOS-agarose-CaCl2 (PTAgC) biocomposite cryogels J Mater Sci Mater Med. 2014; 25(5):1327-37. DOI: 10.1007/s10856-014-5166-8.

24. Ruiz-Ojeda F, Rupérez A, Gomez-Llorente C, et al. Cell Models and Their Application for Studying Adipogenic Differentiation in Relation to Obesity: A Review. Int J Mol Sci. 2016; 17(7):1040. D0I:10.3390/ijms17071040.

25. Dominici M, Le Blanc K, Mueller I, et al. Minimal criteria for defining multipotent mesenchymal stromal cells. The International Society for Cellular Therapy position statement. Cytotherapy. 2006; 8(4):315-7.

26. Suga $H$, Matsumoto D, Eto H, et al. Functional implications of CD34 expression in human adipose-derived stem/progenitor cells. Stem Cells Dev. 2009;18(8):120110. doi: $10.1089 / \mathrm{scd} .2009 .0003$.

27. Rojas-Ríos $P$, González-Reyes A. Concise review: The plasticity of stem cell niches: a general property behind tissue homeostasis and repair. Stem Cells. 2014; 32(4):852-9. DOI: 10.1002/stem.1621. 
28. Donnelly H, Salmeron-Sanchez M, Dalby M. Designing stem cell niches for differentiation and self-renewal. J R Soc Interface. 2018; 15(145):20180388. D0I: 10.1098/rsif.2018.0388.

29. Geckil H, Feng Xu, Xiaohui Zhang, et al. Engineering hydrogels as extracellular matrix mimics. Nanomedicine (Lond). 2010; 5(3):469-484. D0I: 10.2217/nnm.10.12.

30. Santoro M, Marchetti $P$, Rossi F, et al. Smart approach to evaluate drug diffusivity in injectable agar-carbomer hydrogels for drug delivery. J Phys Chem B. 2011; 115(11):2503-10. DOI: 10.1021/jp1111394.

31. Bruggeman KF, Williams RJ, Nisbet DR. Dynamic and Responsive Growth Factor Delivery from Electrospun and Hydrogel Tissue Engineering Materials. Adv Healthc Mater. 2018; 7(1). DOI: 10.1002/adhm.201700836.

32. Tong Z, Solanki A, Hamilos A. Application of biomaterials to advance induced pluripotent stem cell research and therapy. EMBO J. 2015; 34(8): $987-1008$. D0I: 10.15252/embj.201490756.

33. Jha A, Tharp K, Ye J, et al. Enhanced Survival and Engraftment of Transplanted Stem Cells using Growth Factor Sequestering Hydrogels. Biomaterials. 2015; 47: 1-12. DOI: 10.1016/j.biomaterials.2014.12.043.

34. Parker J, Mitrousis N, Shoichet MS. Hydrogel for Simultaneous Tunable Growth Factor Delivery and Enhanced Viability of Encapsulated Cells in Vitro. Biomacromolecules. 2016; 17(2):476-84. D0l: 10.1021/acs.biomac.5b01366.

35. Gothard D, Smith E, Kanczler J. In Vivo Assessment of Bone Regeneration in Alginate/Bone ECM Hydrogels with Incorporated Skeletal Stem Cells and Single Growth Factors. PLoS One. 2015; 10(12):0145080. D0I:10.1371/journal.pone.0145080.

36. Kuchuk O, Kyryk V. Stepwise Differentiation of Multipotent Cells from Murine Adipose Tissue in Osteogenic Direction. Problems of Cryobiology and Cryomedicine. 2012; 22(2):161-164.

37. Fröhlich $M$, Grayson WL, Marolt D. Bone grafts engineered from human adipose-derived stem cells in perfusion bioreactor culture. Tissue Eng Part A. 2010; 16(1):179-89. DOI: 10.1089/ten.TEA.2009.0164.

38. Burdick J, Mauck R, Gerecht S. To Serve and Protect: Hydrogels to Improve Stem Cell-Based Therapies. Cell Stem Cell. 2016; 18(1):13-5. D0I: 10.1016/j. stem.2015.12.004.

39. Negoro T, Takagaki Y, Okura H, Matsuyama A. Trends in clinical trials for articular cartilage repair by cell therapy. NPJ Regen Med. $2018 ; 3: 17$.

40. Webber M, Khan 0, Sydlik S. A Perspective on the Clinical Translation of Scaffolds for Tissue Engineering Ann Biomed Eng. 2015; 43(3):641-656. D0I:10.1007/ s10439-014-1104-7.

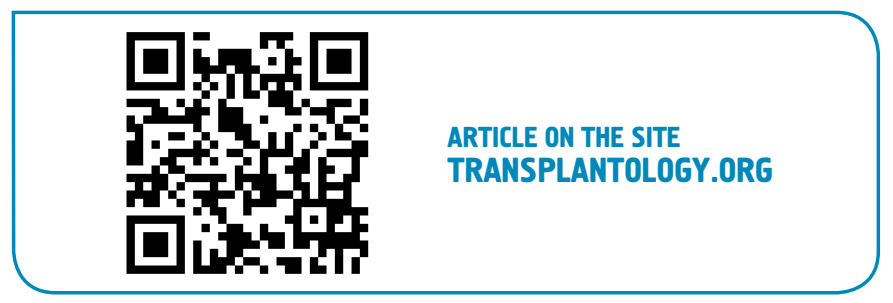

The authors indicate no potential conflicts of interest.

Received: June 13, 2018

Accepted: November 30, 2018 